\title{
The changing face of acute myeloid leukemia therapeutics in the elderly population
}

Acute myeloid leukemia (AML) is primarily a disease of the elderly. $66 \%$ of patients with newly diagnosed AML in the United States are 65 years and older. ${ }^{[1-3]}$ Elderly patients ( $\geq 60-65$ years) with AML have a poor prognosis attributable to having disease that is inherently more resistant to current standard cytotoxic agents and/or relatively poor tolerance of these agents. ${ }^{[4-6]}$ Furthermore, elderly patients with AML more frequently have an antecedent hematological disorder, unfavorable cytogenetics, and poorer performance status at presentation ${ }^{[5,7]}$ As a result, despite steady progress in the therapy of AML in younger patients, the treatment of elderly AML has not improved significantly over the last four decades. ${ }^{[3,8,9]}$ The $4-8$ weeks mortality with intensive chemotherapy is $30-50 \%$ in these patients, and the median survival is 4-7 months.

The poor historical outcomes with intensive chemotherapy have resulted in reluctance by physicians to treat elderly patients with AML. A review of the Surveillance, Epidemiology and End Results and Medicare databases revealed that only $33 \%$ of elderly AML patients received leukemia directed treatment. ${ }^{[10]}$ Median overall survival (OS) for the entire group was 2.5 months. Median OS for treated patients was 6 months longer than for untreated patients. Burnett et al. reported that low-dose cytarabine (LDAC) was associated with a higher complete remission (CR) rate (18\% vs. $1 \%$, $P<0.001$ ) and improved OS (estimated 1-year survival rate, $25 \%$ vs. $5 \% ; P<0.001)$ in elderly AML patients ${ }^{[11]}$. These results highlight the poor outcomes in general, but the potential benefit with leukemia-directed treatment rather than palliation in elderly AML patients, but also the pressing need to develop novel therapeutic strategies better suited for this patient population. ${ }^{[5]}$ A number of these novel agents are currently being evaluated in ongoing clinical trials including the hypomethylating agents (decitabine, azacytidine, SGI-110), purine analogues (clofarabine, cladribine), vosaroxin, CPX351, volasertib, hedgehog inhibitors (PF-04449913, vismodegib), and pracinostat.

Hypomethylating agents are the most frequently used agents in the therapy of elderly AML in the US and Europe. ${ }^{[12]}$ The DACO-016 study compared the efficacy and safety of decitabine $\left(20 \mathrm{mg} / \mathrm{m}^{2} /\right.$ day for 5 days every 4 -week) versus investigators choice (including LDAC $20 \mathrm{mg} / \mathrm{m}^{2} /$ day for 10 days every 4-week or best supportive care) in 485 AML patients (median age 73 years) ineligible for cytotoxic chemotherapy. ${ }^{[12,13]}$ The initial analysis showed a trend toward improved survival with decitabine (7.7 vs. 5.0 months; $P=0.108)$ that became significant $(P=0.037)$ with further follow-up. Azacitidine has been explored in elderly patients with AML with $20-30 \%$ blasts in a subset analysis of the

Access this article online Quick Response Code: 口籍证品

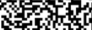

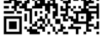

Website: www.sajc.org DOI: $10.4103 / 2278-330 X .149900$ phase III AZA-001 trial. ${ }^{[14]}$ Elderly AML patients (median age 75 years) were randomized to receive either azacitidine ( $75 \mathrm{mg} / \mathrm{m}^{2} /$ day for 7 days every 4 -week) or conventional care regimen (CCR; best supportive care, LDAC $40 \mathrm{mg}$ /day for 10 days every 4 -week or investigators choice). ${ }^{[15]}$ Median OS was 10.4 months (1-year survival $47 \%$ ) for patients receiving azacitidine compared to 6.5 months (1-year survival 34\%) for patients receiving $\operatorname{CCR}(P=0.083)$. SGI-110 is a second-generation hypomethylating agent with a longer half-life and more potent hypomethylation than first-generation hypomethylators. In a phase II study SGI-110 a CR rate of 53\% was reported in treatment-naïve elderly patients not suitable for intensive chemotherapy. ${ }^{[16]}$

Purine analogs have shown encouraging results as single-agents or in combination with LDAC. We have previously demonstrated that the combination of clofarabine and LDAC achieves high response rates with low induction mortality in elderly patients with previously untreated AML. ${ }^{[17]}$ Similarly, cladribine and LDAC alternating with decitabine has been well tolerated with no 4 -week mortality, a CR rate of $58 \%$, and a 1 -year OS rate of $51 \%{ }^{[18]}$

A number of novel therapeutic agents are currently being evaluated in elderly patients with AML (>60-65 years). These include volasertib (a Polo-like kinase 1 inhibitor), vosaroxin (a quinolone derivative topoisomerase II inhibitor with reduced cardiotoxocity), CPX351 (a liposomal formulation of cytarabine and daunorubicin at a fixed molar ratio), PF-04449913 (a small molecule inhibitor of the Sonic Hedgehog Pathway) and pracinostat (a pan-histone deacetylase inhibitor). These drugs are currently in phase II/III studies either as single agents or in combination with hypomethylators or LDAC.

In addition to traditional risk factors such as age, cytogenetics, and performance status, factors such as molecular mutations have prognostic and therapeutic impact in AML. A number of mutated or deregulated genes conferring unfavorable (FLT3-ITD, IDH1/IDH2, WT1, MLL-PTD, TP53, KIT, EVI1, ERG, BAALC), indeterminate (NRAS, KRAS, RUNX1, JAK2, TET2, ASXL1, CBL) or favorable (NPM1, $C E B P A 1, G A T A 2)$ prognosis have been identified. ${ }^{[19,20]}$ In addition to their prognostic value, these mutations offer potential therapeutic targets. A number of clinically active agents targeting FLT3 ITD and/or D835 (such as quizartinib, crenolanib and sorafenib), MEK (activated in patients with $N R A S / K R A S$ mutations) (such as GSK1120212 and MEK-162) and IDH1/IDH2 (such as ABT199 and AG221) are being investigated in AML. These agents are being used as either single-agents or in combination regimens. In many circumstances, the response rates with such targeted therapies are superior to those achieved with standard therapy with decreased toxicity.

Despite the lack of progress in the past decades that has led to a nihilistic approach to treatment of older patients with AML, significant progress is being made recently in the understanding of the biology and development of therapeutic options that offer a brighter future for this large subset of patients with AML.

Naval Daver, Jorge Cortes

Department of Leukemia, The University of Texas MD Anderson Cancer Center, Houston, Texas, USA. Correspondence to: Dr. Naval Daver, E-mail:ndaver@mdanderson.org 


\section{References}

1. Jemal A, Siegel R, Ward E, Murray T, Xu J, Smigal C, et al. Cancer statistics, 2006. CA Cancer J Clin 2006;56:106-30.

2. Löwenberg B, Downing JR, Burnett A. Acute myeloid leukemia. N Engl J Med 1999;341:1051-62.

3. Juliusson G, Antunovic P, Derolf A, Lehmann S, Möllgård L, Stockelberg D, et al. Age and acute myeloid leukemia: Real world data on decision to treat and outcomes from the Swedish Acute Leukemia Registry. Blood 2009;113:4179-87.

4. Kantarjian HM. Therapy for elderly patients with acute myeloid leukemia: A problem in search of solutions. Cancer 2007; 109:1007-10.

5. Kantarjian H, O'brien S, Cortes J, Giles F, Faderl S, Jabbour E, et al. Results of intensive chemotherapy in 998 patients age 65 years or older with acute myeloid leukemia or high-risk myelodysplastic syndrome: Predictive prognostic models for outcome. Cancer 2006;106:1090-8.

6. Nazha A, Ravandi F. Acute myeloid leukemia in the elderly: Do we know who should be treated and how? Leuk Lymphoma 2014;55:979-87.

7. Appelbaum FR, Gundacker H, Head DR, Slovak ML, Willman CL, Godwin JE, et al. Age and acute myeloid leukemia. Blood 2006;107:3481-5.

8. Büchner T, Berdel WE, Haferlach C, Haferlach T, Schnittger S, Müller-Tidow $\mathrm{C}$, et al. Age-related risk profile and chemotherapy dose response in acute myeloid leukemia: A study by the German Acute Myeloid Leukemia Cooperative Group. J Clin Oncol 2009;27:61-9.

9. Kantarjian H, Ravandi F, O'Brien S, Cortes J, Faderl S, Garcia-Manero G, et al. Intensive chemotherapy does not benefit most older patients (age 70 years or older) with acute myeloid leukemia. Blood 2010;116:4422-9.

10. Menzin J, Lang K, Earle CC, Kerney D, Mallick R. The outcomes and costs of acute myeloid leukemia among the elderly. Arch Intern Med 2002; 162: 1597-603.

11 Burnett AK, Milligan D, Prentice AG, et al. A comparison of low-dose cytarabine and hydroxyurea with or without all-trans retinoic acid for acute myeloid leukemia and high-risk myelodysplastic syndrome in patients not considered fit for intensive treatment. Cancer. 2007; 109: 1114-24.

12. Kantarjian HM, Thomas XG, Dmoszynska A, Wierzbowska A, Mazur G, Mayer J, et al. Multicenter, randomized, open-label, phase III trial of decitabine versus patient choice, with physician advice, of either supportive care or low-dose cytarabine for the treatment of older patients with newly diagnosed acute myeloid leukemia. J Clin Oncol 2012;30:2670-7.

13. Thomas XG, Arthur C, Delaunay J, Jones M, Berrak E, Kantarjian HM.
A post hoc sensitivity analysis of survival probabilities in a multinational phase III trial of decitabine in older patients with newly diagnosed acute myeloid leukemia. Clin Lymphoma Myeloma Leuk 2014;14:68-72.

14. Fenaux P, Mufti GJ, Hellström-Lindberg E, Santini V, Gattermann N, Germing $U$, et al. Azacitidine prolongs overall survival compared with conventional care regimens in elderly patients with low bone marrow blast count acute myeloid leukemia. J Clin Oncol 2010;28:562-9.

15. Dombret JF, Butrym A, Wierzbowska A, Selleslag D, Jang RK, Cavenagh J, et al. Results of a phase 3, multicenter, randomized, open-label study of azacytidine (AZA) vs conventional care regimens (CCR) in older patients with newly diagnosed acute myeloid leukemia (AML). Haematologica; Milan: European Hematology Association Annual Meeting Abstract; 2014. p. 788-9.

16. Kantarjian HM, Jabbour E, Yee K, Kropf P, O'Connell C, Stock W, et al. First Clinical results of a randomized phase 2 Study Of SGI-110, a Novel Subcutaneous (SQ) Hypomethylating Agent (HMA), in Adult Patients With Acute Myeloid Leukemia (AML). Blood; New Orleans: American Society of Hematology Annual Meeting 2013, Abstract 122.

17. Parikh SA, Kantarjian H, Garcia-Manero G, Jabbour E, Kadia T, Ravandi F, et al. Clofarabine plus low-dose cytarabine induction followed by consolidation with clofarabine plus low-dose cytarabine alternating with decitabine as frontline therapy for patients (pts) with Acute Myeloid Leukemia $(A M L)>=60$ years (yrs). Blood; New Orleans: American Society of Hematology Annual Meeting 2009, Abstract 114

18. Kadia TM, Borthakur G, Ferrajoli A, Jain P, Jabbour E, Verstovsek S, et al. Phase II Trial Of Cladribine and Low-Dose AraC (LDAC) Alternating with decitabine in older patients with Acute Myeloid Leukemia (AML). Blood; New Orleans: American Society of Hematology Annual Meeting 2013, Abstract 122.

19. Marcucci G, Haferlach T, Döhner H. Molecular genetics of adult acute myeloid leukemia: Prognostic and therapeutic implications. J Clin Oncol 2011;29:475-86.

20. Rockova V, Abbas S, Wouters BJ, Erpelinck CA, Beverloo HB, Delwel R, et al. Risk stratification of intermediate-risk acute myeloid leukemia: Integrative analysis of a multitude of gene mutation and gene expression markers. Blood 2011;118:1069-76.

How to cite this article: Daver N, Cortes J. The changing face of acute myeloid leukemia therapeutics in the elderly population. South Asian J Cancer 2015;4:1-2. Source of Support: Nil. Conflict of Interest: None declared. 